\title{
Propiedades psicométricas y estandarización de la Escala de Ansiedad Cognitiva frente a los Exámenes a la población universitaria argentina.
}

\author{
Luis Furlan*1, Edgardo Pérez*, Marcos Moyano* \& Jerrell Cassady** \\ * Laboratorio de Evaluación Psicológica y Educativa. Universidad Nacional de Córdoba. Argentina. \\ ** Ball State University. Muncie. Indiana. U.S.A.
}

\begin{abstract}
Resumen: En este artículo se presenta una versión adaptada a la población argentina de la Cognitive Test Anxiety Scale (Cassady \& Johnson, 2002), un autoinforme que mide manifestaciones cognitivas de ansiedad frente a los exámenes en estudiantes universitarios. Los análisis factoriales de tipo exploratorio y confirmatorio realizados sugieren una estructura unidimensional de 16 ítems, con elevada consistencia interna y aceptable estabilidad temporal. Las evidencias de validez basada en criterio, respecto a rendimiento académico, perfeccionismo, autorregulación atencional y pensamientos automáticos negativos son teóricamente congruentes. Por otra parte, se elaboraron normas de interpretación diferenciadas por sexo, lo que facilita el empleo del instrumento con fines epidemiológicos y de diagnóstico temprano de estudiantes con elevada ansiedad frente a los exámenes. Finalmente, contar con una escala equivalente a la versión original en inglés facilita la realización de estudios transculturales.
\end{abstract}

Palabras clave: Ansiedad Frente a los Exámenes; Validez; Normas de Interpretación.

Title: Psychometric Properties and standardization of the Cognitive Test Anxiety Scale to argentinean university population.

Abstract: In this article we present an adapted to Spanish version of Cognitive Test Anxiety Scale, a self report measuring cognitive manifestations of test anxiety for university students. Exploratory and confirmatory factor analyses suggest an unidimentional 16 items structure, with high internal consistence and moderate temporal stability. Test - criteria validity evidences, regard to academic performance, perfectionism, attentional self- regulation and automatic negative thoughts are theoretically congruent and provide aditionally support. Moreover, normative values by each sex were elaborated, to facilitate the use of the scale with epidemiological purposes and earlier detection of test anxious students. Finally, have a scale equivalent to original English version facilitate the development of trascultural studies.

Key Words: Test Anxiety; Validity; Norms of Interpretation.

\section{Introducción}

La ansiedad ante los exámenes ("test anxiety"; Mandler \& Sarason, 1952) es un constructo que ha sido investigado sistemáticamente desde hace siete décadas. Puede definirse como una reacción emocional que las personas presentan frente a una situación en la cual sus aptitudes son evaluadas. Una característica definitoria es la preocupación recurrente por el posible fracaso o mal rendimiento en la tarea, la cual posee consecuencias aversivas en la autoestima, minusvaloración social, y pérdida de algún beneficio esperado (Gutiérrez Calvo \& Avero 1995).

\footnotetext{
${ }^{1}$ Por favor dirigir la correspondencia relacionada con este artículo a: Luis Furlan, E-mail: furlan@psyche.unc.edu.ar
} 
La delimitación de este concepto ha experimentado sucesivas transformaciones que se reflejan en los diferentes instrumentos construidos para su medición. Desde una primera perspectiva de la Ansiedad frente a los Exámenes como respuesta global y unitaria (Mandler \& Sarason, 1952) se evolucionó hacia concepciones primero bidimensionales (Sarason, 1978; Spielberger, 1980) y luego multidimensionales (Ferrando, Varea \& Lorenzo, 1999; Sarason, 1984; Hoddap, 1996), apoyadas en propuestas teóricas innovadoras y hallazgos empíricos obtenidos mediante el empleo de análisis factorial exploratorio y confirmatorio.

Furlan (2006) realizó una exhaustiva revisión de los instrumentos para medir ansiedad frente a los exámenes informados en la literatura, concluyendo que se requería adaptar o construir una escala para su uso en estudiantes argentinos, dada la inexistencia de un instrumento aplicable a población hispana que acreditara evidencias satisfactorias de su validez, particularmente en lo relativo a su estructura interna. Como producto de esta revisión se seleccionó la "Cognitive Test Anxiety Scale" CTAS (Cassady \& Johnson, 2002) para realizar su adaptación a la población universitaria argentina, en colaboración con su autor (Furlan, Cassady \& Pérez, 2009).

Esta escala fue elegida por diferentes razones. En primer lugar, existe consenso entre los investigadores respecto a que el componente cognitivo de la respuesta ansiosa, tradicionalmente denominado preocupación (worry), es el que se correlaciona negativamente y de forma estadísticamente significativa con el rendimiento académico (Hembree, 1988; Zeidner, 1998). Sin embargo, luego de un análisis pormenorizado de los indicadores que se agrupan bajo este concepto en las diversas escalas existentes se advirtió la presencia de ítems referentes a procesos cognitivos diferentes a la preocupación, tales como la interferencia, los pensamientos no relacionados a la tarea y creencias autoderrotistas. Por ello se estimó que "cognitive test anxiety", denominación propuesta por Cassady y Johnson (2002), aparecía como más ajustada, para dar cuenta de dicha diversidad de manifestaciones cognitivas, que conforman uno de los principales componentes de la respuesta ansiosa frente a los exámenes.

Adicionalmente, se consideraron las propiedades satisfactorias de la escala en cuanto a consistencia interna, evidencias de validez externa con relación a rendimiento académico, además de su extensión breve y pertinencia en relación a los intereses del equipo de investigación. Los ítems de la versión original estaban publicados y el autor principal brindó su acuerdo y participó activamente en el proceso de adaptación. 
En su versión original, la Cognitive Test Anxiety Scale (Cassady \& Johnson, 2002) posee 27 ítems con un formato de respuesta likert de 4 puntos que van desde 1 (nada frecuente en mi) a 4 (muy frecuente en mi). Esta escala evalúa la tendencia a preocuparse por un eventual mal rendimiento, poseer pensamientos irrelevantes en cuanto a la tarea durante el examen y en los períodos de preparación del mismo, realizar comparaciones con otros pares mientras se está rindiendo un examen o preparando para el mismo, presencia de pensamientos intrusivos en las evaluaciones y las sesiones de estudio, y pensamientos de escape que interfieren con la atención mientras se rinde. Los estudios previos de estructura interna indicaron que la escala era unidimensional y con elevada consistencia interna $(\alpha=.91)$.

\section{Adaptación al español}

Para su adaptación se realizó una traducción inversa de los ítems y se verificó la equivalencia entre la versión original y traducida. La versión en español se administró a una muestra reducida de estudiantes para evaluar la comprensión de los ítems, realizando pequeños ajustes de fraseo en algunos de ellos. Posteriormente, se llevaron a cabo estudios de estructura interna, para los cuales se administró la escala a 752 estudiantes de Química (60\%) y Psicología (40\%).

Esta muestra fue dividida aleatoriamente en dos partes y con la primera de ellas se llevó a cabo un análisis factorial exploratorio (Máxima Verosimilitud y rotación Promax) que arrojó una primer solución con dos factores, explicando el $35,83 \%$ de la varianza. No obstante, el segundo factor resultó problemático de interpretar, y se atribuyó la conformación del mismo a un efecto de la codificación de los ítems, que en su totalidad eran de sentido inverso e indicadores de baja ansiedad. (DiSteffano \& Motl, 2006). Con los 18 ítems de codificación directa se realizó un nuevo análisis factorial, obteniendo un único factor, que explicó el 29.88\% de la varianza $(\alpha=.88)$. Dos ítems se eliminaron por presentar correlaciones ítem factor menores a .30. Esta versión abreviada presentó una elevada correlación $(\mathrm{r}=.94, \mathrm{p}$ $<.0001$ ) con la forma original de 27 ítems, lo que sugiere la factibilidad de evaluar el mismo constructo mediante una escala más breve y de estructura interna más clara. En la segunda mitad de la muestra se realizó un análisis factorial confirmatorio, con el objetivo de poner a prueba el modelo de 16 ítems y un factor y comparar sus índices de ajuste con dos modelos alternativos, uno de dos factores y otro unidimensional de mayor cantidad de ítems. Los resultados mostraron un mejor ajuste del modelo de un factor y 16 ítems. La escala obtenida 
se denominó S-CTAS (Spanish Cognitive Test Anxiety Scale), y evidenció un índice adecuado de estabilidad en un intervalo de 90 días $(\mathrm{r}=.768, \mathrm{p}<.01$; Furlan, et al. 2009).

La S-CTAS ha sido utilizada en diferentes investigaciones, permitiendo obtener información empírica sobre sus relaciones con otras variables. Como evidencia de las relaciones test-criterio respecto del rendimiento académico, en un estudio reciente realizado en una muestra de 723 estudiantes universitarios se obtuvieron correlaciones moderadas negativas $r=-.34, p<.01$ con el promedio de calificaciones autoinformado (Moyano, 2010), en concordancia con lo que diferentes autores han señalado previamente (Cassady \& Johnson, 2002; Gutiérrez \& Calvo, 1996; Hembree, 1988). En el mismo estudio, un análisis de varianza arrojó diferencias estadísticamente significativas en los puntajes de la S-CTAS entre grupos de estudiantes perfeccionistas no adaptativos, adaptativos y no perfeccionistas. La dimensión Discrepancia del Perfeccionismo (Arana, Keegan \& Rusztein, 2009) presentó una correlación positiva, estadísticamente significativa y grande $(\mathrm{r}=.527, \mathrm{p}<.001)$ con la $\mathrm{S}$ CTAS. En el mismo sentido se obtuvieron correlaciones moderadas $(r=.389, \mathrm{p}<.001)$ con una escala de Pensamientos Automáticos Negativos (Moyano, 2010). Furlan, Kohan Cortada, Piemontesi y Heredia (2008) en una muestra de 188 estudiantes universitarios de Buenos Aires, analizaron las relaciones de la ansiedad cognitiva frente a los exámenes y la auto regulación de la atención, obteniendo una correlación negativa y moderada $(\mathrm{r}=-.361, \mathrm{p}<$. 001) entre la S-CTAS y la Self-Regulation Scale (Schwarzer, Diehl \& Schmitz, 1999).

Como evidencia de validez relacionada con grupos contrastados, se analizaron en diferentes oportunidades las diferencias de género. En un primer estudio (Furlan et al., 2009) se obtuvo un valor $\mathrm{t}(269)=2.49, \mathrm{p}<.013$, indicando una diferencia pequeña y significativa, donde las mujeres $(\mathrm{M}=35.25, \mathrm{ds}=9.14)$ informaron mayores niveles de ansiedad cognitiva que los varones $(\mathrm{M}=32.60, \mathrm{ds}=9.40)$. Posteriormente, Moyano (2010) obtuvo resultados semejantes, con un valor $\mathrm{t}(725)=-2,51 \mathrm{p}<.001$, con puntajes para el grupo femenino $(\mathrm{M}=$ 36.46 , ds $=9.89)$ mayores que en el masculino $(\mathrm{M}=33.95$, ds $=8.10)$. A partir de las evidencias obtenidas, se puede afirmar que esta escala ha dado muestras convincentes de su utilidad para medir ansiedad frente a los exámenes en población argentina y que su versión en español puede ser utilizada con fines de investigación así como de diagnóstico e identificación de estudiantes en situación de riesgo académico. Como un objetivo específico de este trabajo se plantea la necesidad de contar con normas de interpretación locales. 


\section{Método}

\subsection{Participantes.}

Se conformó una muestra accidental de $\mathrm{N}=795$ estudiantes (femenino $=73 \%$, masculino $=26 \%$ ) de 40 diferentes carreras de la U.N.C. Los alumnos cursaban desde el primero hasta el último año de la carrera. La media de edad fue de 23.8 años (D.S $=5.15$, rango $=17-58)$.

\subsection{Instrumentos.}

Spanish Cognitive Test Anxiety Scale (S-CTAS): es una escala de 16 ítems con cuatro alternativas de respuesta (1- nada frecuente en mi a 4- muy frecuente en mí). Las puntuaciones se obtienen sumando las respuestas a la totalidad de los ítems y oscilan entre 16 y 64. La consistencia interna de la escala es de $(\alpha=.88)$.

\subsection{Procedimiento.}

Se realizó un estudio de tipo instrumental (Montero \& León, 2007) orientado a la elaboración de normas de interpretación de la S-CTAS. Para la recolección de datos se creó una página Web en donde estaba a disposición de los participantes la escala para ser completada y enviada directamente a una base de datos generada en el programa estadístico Excel. Luego, en diciembre de 2009 se realizaron las invitaciones a los estudiantes (mediante mailing, Facebook, comentarios personales, etc.) para participar del estudio propiamente dicho. A los participantes se les generaba automáticamente un informe con los puntajes obtenidos y algunos comentarios acerca de su significado. Los datos fueron procesados con el paquete estadístico SPSS -11 .

\section{Resultados}

Se calcularon puntajes $\mathrm{T}$ diferenciados para varones y mujeres, atendiendo a que los estudios realizados previamente, indicaron de manera consistente diferencias significativas en los puntajes promedio de Ansiedad frente a los Exámenes de acuerdo al género. Para el establecimiento de los puntos de corte a partir de los cuales se considera la condición clínicamente significativa, se calcularon los puntajes $Z$, considerando el límite en $1 \frac{1 / 2}{Z}$ por encima de la media, lo que equivale en la distribución $\mathrm{T}$ diferenciada por género a un valor $\mathrm{T}$ 66. Para este valor corresponde en los varones un puntaje bruto de 47 y para las mujeres de 52. Los sujetos que puntúan por encima de estos valores tienen una elevada probabilidad de 
presentar dificultades emocionales asociadas a las evaluaciones.

Tabla1. Puntajes T de la S-CTAS para la muestra total de estudiantes universitarios diferenciados por género

\begin{tabular}{|c|c|c|c|c|c|}
\hline & $\begin{array}{l}\text { Varones } \\
(\mathrm{N}=210)\end{array}$ & $\begin{array}{c}\text { Mujeres } \\
(\mathrm{N}=585)\end{array}$ & & $\begin{array}{l}\text { Varones } \\
(\mathrm{N}=210)\end{array}$ & $\begin{array}{c}\text { Mujeres } \\
(\mathrm{N}=585)\end{array}$ \\
\hline $\begin{array}{l}\text { Puntajes } \\
\text { brutos }\end{array}$ & $\mathrm{T}$ & $\mathrm{T}$ & $\begin{array}{l}\text { Puntajes } \\
\text { brutos }\end{array}$ & & \\
\hline 16 & 29 & 29 & 41 & 59 & 55 \\
\hline 17 & 30 & 30 & 42 & 60 & 56 \\
\hline 18 & 31 & 31 & 43 & 61 & 57 \\
\hline 19 & 32 & 32 & 44 & 62 & 58 \\
\hline 20 & 33 & 33 & 45 & 64 & 59 \\
\hline 21 & 34 & 34 & 46 & 65 & 60 \\
\hline 22 & 35 & 35 & 47 & 66 & 61 \\
\hline 23 & 36 & 36 & 48 & 67 & 62 \\
\hline 24 & 38 & 37 & 49 & 69 & 63 \\
\hline 25 & 39 & 38 & 50 & 70 & 64 \\
\hline 26 & 40 & 39 & 51 & 71 & 65 \\
\hline 27 & 41 & 40 & 52 & 72 & 66 \\
\hline 28 & 43 & 41 & 53 & 73 & 67 \\
\hline 29 & 44 & 42 & 54 & 75 & 68 \\
\hline 30 & 45 & 43 & 55 & 76 & 69 \\
\hline 31 & 46 & 44 & 56 & 78 & 70 \\
\hline 32 & 48 & 45 & 57 & 79 & 71 \\
\hline 33 & 49 & 46 & 58 & - & 72 \\
\hline 34 & 50 & 47 & 59 & - & 73 \\
\hline 35 & 51 & 49 & 60 & - & 74 \\
\hline 36 & 53 & 50 & 61 & - & 75 \\
\hline 37 & 54 & 51 & 62 & - & 76 \\
\hline 38 & 55 & 52 & 63 & - & 77 \\
\hline 39 & 56 & 53 & 64 & - & 78 \\
\hline 40 & 57 & 54 & & & \\
\hline
\end{tabular}

\section{Discusión}

La adaptación y estandarización de una escala como la aquí presentada ha requerido un trabajo colaborativo y cuidadoso, para lograr una versión del instrumento equivalente a la original con propiedades psicométricas adecuadas. El proceso realizado permitió obtener una escala más breve que la original y con una estructura interna más clara, manteniendo una elevada consistencia interna. Según el autor de la versión original (Cassady, comunicación personal, 2009) los patrones de relación de la versión original de la CTAS (27 items) con otras variables, investigados previamente (Cassady \& Johnson, 2002; Cassady, 2004), se mantienen muy similares al volver a computarlos con la versión abreviada, lo que brinda 
apoyo adicional al trabajo realizado y confirma su utilidad.

Hasta ahora la S-CTAS ha sido empleada en estudios de tipo ex post facto de moderada complejidad, mayormente conducidos por nuestro equipo y resultaría de gran valor, conocer el funcionamiento de la escala en estudios que utilicen análisis multivariados y modelos de ecuaciones estructurales, similares a los que se han realizado en sus contextos originales. También se prevé su utilización como medida pre y post tratamiento en estudios sobre la eficacia de intervenciones terapéuticas para la ansiedad frente a los exámenes. La publicación de la escala en castellano puede incentivar ese uso relevante. Adicionalmente, su utilización en otros países hispano parlantes, especialmente latinoamericanos, permitirá el desarrollo de estudios comparados y subsanará el estado de relativa vacancia de instrumentos adaptados para medir ansiedad frente a los exámenes en estos contextos.

Para su utilización en el ámbito clínico, resultaría provechoso complementarla con escalas que evalúen las dimensiones de emotividad (por ejemplo, el GTAI - A, Heredia, Piemontesi, Furlan \& Hodapp, 2009) y con una entrevista que explore comportamientos disfuncionales asociados (por ejemplo, la procrastinación) y la percepción de los estudiantes respecto de modalidades evaluativas específicas (que la escala no diferencia), a fin de obtener una valoración más integral del problema y planear estrategias específicas de asistencia.

Finalmente, los baremos elaborados para nuestro medio permitirán una interpretación más clara de los puntajes individuales, y el establecimiento de puntos de corte diferenciados para establecer condiciones clínicamente significativas, es decir, puntajes a partir de los cuales, la ansiedad frente a los exámenes aparezca claramente asociada con malestar subjetivo elevado y dificultades académicas.

\section{Referencias}

Arana, F. G., Keegan, E. G., y Rutsztein, G. (2009). Adaptación argentina de una medida multidimensional de perfeccionismo: la Almost Perfect Scale-Revised (APS-R). Evaluar, 9, $35-53$.

Cassady, J. C. (2004). The impact of cognitive test anxiety across the learning testing cycle. Learning and Instruction, 14, 569-592.

Cassady, J. C., \& Johnson, R. E. (2002). Cognitive test anxiety and academic performance. Contemporary Educational Psychology, 27, 270-295. 
DiStefano, C., \& Motl, R.W., (2006). Further investigating method effects associated with negatively worded items on self-report surveys. Structural Equation Modeling, 13(3), 440-464.

Ferrando, P., Verea, M., \& Lorenzo, U. (1999). Evaluación Psicométrica del Cuestionario de Ansiedad y Rendimiento en una muestra de escolares. Psicothema, 11(1), 225-236.

Furlan, L. (2006) Ansiedad ante a los exámenes: Qué y cómo se evalúa? Evaluar, 6, 32-51.

Furlan, L., Kohan Cortada, A., Piemontesi, S., y Heredia, D. (2008). Autorregulación de la Atención, Afrontamiento y Ansiedad ante los Exámenes en estudiantes universitarios. Jornadas de investigación, Facultad de Psicología Universidad de Buenos Aires. Buenos Aires.

Furlan, L. A., Cassady, J. C., \& Pérez, E. R. (2009). Adapting the Cognitive Test Anxiety Scale for use with argentinean university students. Internacional Journal of Testing, 9(1), 3-13.

Gutiérrez Calvo, M., \& Avero, P. (1995). Ansiedad, estrategias auxiliares y comprensión lectora: déficit de procesamiento vs falta de confianza. Psicothema, 7(3), 569-578.

Gutiérrez Calvo, M. (1996). Ansiedad y Deterioro Cognitivo: Incidencia en el Rendimiento académico Ansiedad y Estrés, 2(2-3), 173-194.

Hembree, H. (1988). Correlates, causes, effects, and treatment of test anxiety. Review of Educational Research, 58, 47-77.

Heredia, D.; Piemontesi, S., Furlan, L. \& Hodapp, V. (2008). Adaptación del Inventario Alemán de Ansiedad frente a los Exámenes (GTAI-A). Evaluar 8, 46-60.

Hodapp, V. (1996). The TAI-G: A multidimensional approach to the assessment of test anxiety. In C. Schwarzer \& M. Zeidner (Eds.), Stress, Anxiety, and Coping in Academic Settings, pp. 95130. Tübingen: Francke.

Mandler, G. \& Sarason, S.B. (1952). A study of anxiety and learning. Journal of Abnormal and Social Psychology, 47, 166-173.

Montero I. y León O. G. (2007) A guide for naming research studies in pshychology. [Guía para nombrar estudios de investigación en psicología] International Journal of Clinical and Health Psychology, 7(3), 847-862.

Moyano, M. (2010) Ansiedad ante los Exámenes, Pensamientos Automáticos Negativos y Perfeccionismo en Estudiantes de la Universidad Nacional de Córdoba. Trabajo Final de Licenciatura. Inédito. Facultad de Psicologia, Universidad Nacional de Córdoba.

Sarason, I.G. (1978) The Test Anxiety Scale: concept and research. In CD Spielberger \& I.G. Sarason (Eds) Stress and Anxiety (Vol.5, pp 193-216). Washington DC: Hemisphere.

Sarason, I.G. (1984) Stress, anxiety, and cognitive interference: Reactions to tests. Journal of Personality and Social Psychology, 46, 929-938.

Schwarzer, R., Diehl, M., \& Schmitz, G. S. (1999). Self-Regulation Scale. Recuperado el 28 de Octubre 28 de 2007, de http://www.fu-berlin.de/gesund/skalen. 
Spielberger, C.D., Gonzalez, H.P., Taylor, C.J., Anton, W.D., Algaze, B., Ross, G.R. \& Westberry, L.G. (1980). Test Anxiety Inventory. Palo Alto, CA: Consulting Psychologists Press.

Zeidner, M. (1998). Test anxiety: the state of the art. New York and London: Plenum Press. 


\section{Anexo 1}

\section{S-CTAS Escala de Ansiedad Cognitiva frente a los exámenes}

Las siguientes afirmaciones describen experiencias de los estudiantes universitarios en relación a los exámenes. Indica cuan habituales resultan para vos usando la siguiente escala: 1="Nada frecuente en mi $2=$ algo frecuente en mi $3=$ bastante frecuente en mi, 4 ="muy frecuente en mi"

1. La preocupación por los exámenes me quita el sueño.

2. Cuando tengo que enfrentar exámenes finales me bloqueo.

3. Durante los exámenes pienso mucho en las consecuencias de reprobar.

4. Al comenzar un examen estoy tan nervioso que a menudo no puedo pensar con claridad.

5. Cuando en un examen estoy presionado por responder, mi mente se pone en blanco.

6. Durante los exámenes muchas veces suelo pensar que tal vez no soy muy brillante.

7. Durante los exámenes me pongo tan nervioso que olvido cosas que realmente conozco.

8. Después de rendir un examen siento que podría haberlo hecho mejor.

9. Realizar bien los exámenes me preocupa más de lo que debería.

10. Durante los exámenes tengo la sensación de que no me está yendo bien.

11. Cuando rindo un examen difícil me siento derrotado aún antes de comenzar.

12. En los exámenes no demuestro todo lo que se acerca de un tema.

13. No soy bueno para rendir exámenes.

14. Cuando me entregan un examen demoro un tiempo para calmarme y pensar con claridad.

15. En los exámenes no obtengo buenos resultados.

16. Cuando rindo un examen me pongo tan nervioso que cometo errores tontos. 